DO-TH 93-08

SNUTP 91-12

YUMS 93-05

(March 1993)

\title{
Flavor Democracy in Standard Models at High Energies
}

\author{
G. Cvetič \\ Inst. für Physik, Universität Dortmund, 4600 Dortmund 50, Germany \\ C. S. Kim \\ Dept. of Physics, Yonsei University, Seoul 120-749, Korea
}

\begin{abstract}
It is possible that the standard model (SM) is replaced around some transition energy $\Lambda$ by a new, possibly Higgsless, "flavor gauge theory" such that the Yukawa (running) parameters of SM at $E \sim \Lambda$ show up an (approximate) flavor democracy (FD). We investigate the latter possibility by studying the renormalization group equations for the Yukawa couplings of SM with one and two Higgs doublets, by evolving them from given physical values at low energies $(E \simeq 1 G e V)$ to $\Lambda\left(\sim \Lambda_{\text {pole }}\right)$ and comparing the resulting fermion masses and CKM matrix elements at $E \simeq \Lambda$ for various $m_{t}^{p h y}$ and ratios $v_{u} / v_{d}$ of vacuum expectation values. We find that the minimal SM and the closely related SM with two Higgs doublets (type I) show increasing deviation from FD when energy is increased, but that SM with two Higgs doublets (type II) clearly tends to FD with increasing energy - in both the quark and the leptonic sector (q-q and l-l FD). Furthermore, we find within the type II model that, for $\Lambda_{\text {pole }} \ll \Lambda_{\text {Planck }}, m_{t}^{\text {phy }}$ can be less than $200 \mathrm{GeV}$ in most cases of chosen $v_{u} / v_{d}$. Under the assumption that also the corresponding Yukawa couplings in the quark and the leptonic sector at $E \simeq \Lambda$ are equal (lq FD), we derive estimates of bounds on masses of top quark and tau-neutrino, which are compatible with experimental bounds.
\end{abstract}




\section{Introduction}

The hierarchical pattern of the quark masses, their mixing, and the Higgs sector in general, remain outstanding issues of the Standard model (SM) electroweak theory. While a gauge interaction is characterized by its universal coupling constant, the Yukawa interactions may have as many coupling constants as there are pairs of fermionic fields coupled to neutral Higgs bosons. There is no apparent underlying principle which governs the hierarchy of the various Yukawa couplings. As a result, SM predicts neither the fermion masses nor their mixing.

For this reason, we regard it as possible that around some high energy $\Lambda$ (>1TeV) SM breaks down and is replaced there by a new extended gauge theory that is responsible for an (approximate) flavor democracy of SM at high energies near the transition $(E \sim \Lambda)$. Flavor democracy (FD) in a fermionic sector basically means equality of Yukawa couplings in this sector in a flavor basis.

An extended gauge theory responsible for FD we call "flavor gauge theory" (FGT). In Appendix A we outline a possible simplified scheme for such an FGT, in order to motivate the concept of flavor democracy. In Section 2 we introduce formally the concept of FD for various fermionic sectors. In Appendix B, possible parametrizations for the deviations from FD are discussed. In Section 3 we use 1-loop renormalization group equations (RGE's) for Yukawa couplings of quarks in SM with one and two Higgs doublets, by considering the second and the third families of quarks together. We evolve RGE's from the low energy $(\sim 1 G e V)$ physical mass scale to high energy and investigate whether the deviations from FD in the quark (up-type and down-type) sectors decrease with increasing energy. We then investigate the same question also in the leptonic sectors. Search for such trends toward FD can be interpreted also as a somewhat unconventional search (i.e., from the low energy physical scale to high energy) within SM for signals of new physics. In Section 4 we adopt the assumption of an additional lepton-quark FD (i.e., that at high energies the Yukawa couplings in the corresponding quark and leptonic sectors coincide at a high energy $E \simeq \Lambda$ ), which leads to estimates of bounds on masses of the top quark and tau-neutrino. These turn out to be compatible with experimental bounds. Section 5 contains further discussion of results. Conclusions of this paper are summarized in Section 6 . 


\section{Flavor Democracy}

We define that SM at some energy $\mu \simeq \Lambda$ possesses flavor democracy (FD) if there exists a flavor basis 1 where the Yukawa coupling strengths are equal in certain fermionic sectors. In this respect, we will restrict ourselves to two cases of SM, as motivated in Appendix A: the minimal SM, and SM with two Higgs doublets (type II) 迎.

\section{a) Minimal SM:}

FD would mean here an "overall" FD - with equal coupling strength for the up-type and the down-type fermionic sector simultaneously (for quarks, and separately for leptons):

$$
\begin{aligned}
\mathcal{L}_{\text {Yukawa }}(\Lambda) \simeq & -G^{(q)} \sum_{i, j=1}^{3}\left\{\left[\left(\bar{q}_{L}^{(i)} \tilde{H}\right) q_{u R}^{(j)}+\text { h.c. }\right]+\left[\left(\bar{q}_{L}^{(i)} H\right) q_{d R}^{(j)}+\text { h.c. }\right]\right\} \\
& -G^{(l)} \sum_{i, j=1}^{3}\left\{\left[\left(\bar{l}_{L}^{(i)} \tilde{H}\right) l_{u R}^{(j)}+\text { h.c. }\right]+\left[\left(\bar{l}_{L}^{(i)} H\right) l_{d R}^{(j)}+\text { h.c. }\right]\right\},
\end{aligned}
$$

where we use the notation

$$
\begin{gathered}
H=\left(\begin{array}{c}
H^{+} \\
H^{0}
\end{array}\right), \quad \tilde{H}=i \tau_{2} H^{*}, \\
q^{(i)}=\left(\begin{array}{c}
q_{u}^{(i)} \\
q_{d}^{(i)}
\end{array}\right), \quad q^{(1)}=\left(\begin{array}{c}
u \\
d
\end{array}\right), q^{(2)}=\left(\begin{array}{c}
c \\
s
\end{array}\right), q^{(3)}=\left(\begin{array}{c}
t \\
b
\end{array}\right), \\
l^{(i)}=\left(\begin{array}{c}
l_{u}^{(i)} \\
l_{d}^{(i)}
\end{array}\right), \quad l^{(1)}=\left(\begin{array}{c}
\nu_{e} \\
e
\end{array}\right), l^{(2)}=\left(\begin{array}{c}
\nu_{\mu} \\
\mu
\end{array}\right), l^{(3)}=\left(\begin{array}{c}
\nu_{\tau} \\
\tau
\end{array}\right) .
\end{gathered}
$$

\section{b) SM with two Higgs doublets $H^{(u)}$ and $H^{(d)}$ (type II):}

$H^{(u)}$ and $H^{(d)}$ couple in this SM to $f_{u R}$ and $f_{d R}$ fermion isosinglets, respectively [1]. FD would mean here an up-type FD and a separate down-type FD, in the quark and the leptonic sector, i. e. "q-q up-type FD", "q-q down-type FD", "l-l up-type FD" and "l-l down-type FD"

$$
\begin{aligned}
\mathcal{L}_{\text {Yukawa }}(\Lambda) \simeq & -G_{u}^{(q)} \sum_{i, j=1}^{3}\left[\left(\bar{q}_{L}^{(i)} \tilde{H}^{(u)}\right) q_{u R}^{(j)}+\text { h.c. }\right]-G_{d}^{(q)} \sum_{i, j=1}^{3}\left[\left(\bar{q}_{L}^{(i)} H^{(d)}\right) q_{d R}^{(j)}+\text { h.c. }\right] \\
& -G_{u}^{(l)} \sum_{i, j=1}^{3}\left[\left(\bar{l}_{L}^{(i)} \tilde{H}^{(u)}\right) l_{u R}^{(j)}+\text { h.c. }\right]-G_{d}^{(l)} \sum_{i, j=1}^{3}\left[\left(\bar{l}_{L}^{(i)} H^{(d)}\right) l_{d R}^{(j)}+\text { h.c. }\right] .
\end{aligned}
$$

\footnotetext{
${ }^{1}$ Flavor basis is a basis in which the left-handed doublets of fermions transform as doublets under $S U(2)_{L}$.
} 
For the mass matrices $M_{u}^{(q, l)}$ and $M_{d}^{(q, l)}$, FD implies

$$
M_{\alpha}^{(q, l)} \simeq \frac{G_{\alpha}^{(q, l)} v_{\alpha}}{\sqrt{2}}\left[\begin{array}{ccc}
1 & 1 & 1 \\
1 & 1 & 1 \\
1 & 1 & 1
\end{array}\right], \quad(\alpha=u, d)
$$

where $v_{\alpha}=\sqrt{2}\left\langle\left(H^{(\alpha)}\right)^{0}\right\rangle_{o}$ are the vacuum expectation values (VEV). Upon diagonalization, one obtains FD conditions for masses and mixings at $\mu=\Lambda$

$$
M_{\alpha}^{(q, l) d i a g} \simeq \frac{3 G_{\alpha}^{(q, l)} v_{\alpha}}{\sqrt{2}}\left[\begin{array}{ccc}
0 & 0 & 0 \\
0 & 0 & 0 \\
0 & 0 & 1
\end{array}\right], \quad V_{c k m}^{(q, l)} \simeq\left[\begin{array}{ccc}
1 & 0 & 0 \\
0 & 1 & 0 \\
0 & 0 & 1
\end{array}\right]
$$

Note that even at low energies $(\mu \simeq 1-100 \mathrm{GeV})$, the fermionic mass spectrum is rather close to this form - i.e., the deviations from FD in SM in the up-type, and the down-type sectors, are already at low energies not large.

In our calculations, we will approximate the masses of the lightest quark and leptonic family to be zero at energy $\mu_{0}=1 G e V$, and will assume that their mixings to other families $\left(\propto\left(V_{c k m}\right)_{u \beta}, \beta=s, b\right)$ are not strong enough to affect substantially the masses in those families at energies $\mu>\mu_{0}(=1 G e V)$. These assumptions are based on previous investigations of renormalization group equations (RGE's) of SM (e. g. see [2]). Furthermore, first we will restrict our calculations to the quark sector $((c, s)$ and $(t, b))$, since the leptonic sector doesn't affect the evolution of the Yukawa couplings for quarks substantially, as will be seen later. We adopt the light quark masses (at $\mu_{0}=1 \mathrm{GeV}$ ) obtained by Gasser and Leutwyler [3]: $m_{c}=1.35 \pm 0.05 \mathrm{GeV}$, $m_{s}=0.175 \pm 0.05 G e V$ and $m_{b}=5.3 \pm 0.1 \mathrm{GeV}$. For the (physical) mass of the top quark $m_{t}^{p h y}=m_{t}\left(\mu=m_{t}^{p h y}\right)$, we consider the range between $100 \mathrm{GeV}$ and $200 \mathrm{GeV}$, to explore the whole possible region of the experimentally predicted $m_{t}^{p h y}$ 听, 5]. In this case of the two families 2 the Cabibbo-Kobayashi-Maskawa matrix for quarks, at $\mu_{0}=1 \mathrm{GeV}$, is [6]

$$
V_{c k m}^{(q)}\left(\mu=\mu_{0}\right)=\left(\begin{array}{cc}
1-\epsilon^{(q)} & \eta^{(q)} \\
-\eta^{(q)} & 1-\epsilon^{(q)}
\end{array}\right) \simeq\left(\begin{array}{cc}
1-1.25 \times 10^{-3} & 0.05 \\
-0.05 & 1-1.25 \times 10^{-3}
\end{array}\right) .
$$

The matrix is orthonormal, CP-violation effects are neglected.

\footnotetext{
${ }^{2}$ Please note that we are still considering SM with $n_{f}=3$, but the first family being decoupled from the other two in the Yukawa sector.
} 
The trend toward FD, as $\mu \uparrow \Lambda$, would then mean, according to (5) 3

$$
\begin{gathered}
\delta_{u}^{(q)}\left(=\frac{m_{c}}{m_{t}}\right), \delta_{u}^{(l)}\left(=\frac{m_{\nu_{\mu}}^{D}}{m_{\nu_{\tau}}^{D}}\right) \longrightarrow 0, \\
\delta_{d}^{(q)}\left(=\frac{m_{s}}{m_{b}}\right), \delta_{d}^{(l)}\left(=\frac{m_{\mu}}{m_{\tau}}\right) \longrightarrow 0, \\
\eta^{(q)}, \eta^{(l)} \longrightarrow 0 .
\end{gathered}
$$

In the case of the minimal SM ("overall FD") we would have in addition

$$
\frac{m_{b}}{m_{t}}, \frac{m_{\tau}}{m_{\nu_{\tau}}^{D}} \longrightarrow 1
$$

In Appendix B, we show how to find FD-flavor bases, i. e. flavor bases in which we have in the case of FD equal Yukawa coupling strengths in fermionic sectors. There we argue that in an "up-down symmetric" FD-flavor basis the parameters of deviation from FD are

$$
\begin{aligned}
& \triangle_{u}^{(q)}=\sqrt{\left(\frac{m_{c}}{m_{t}}\right)^{2}+\left(\frac{\eta^{(q)}}{2}\right)^{2}}, \\
& \triangle_{d}^{(q)}=\sqrt{\left(\frac{m_{s}}{m_{b}}\right)^{2}+\left(\frac{\eta^{(q)}}{2}\right)^{2}},
\end{aligned}
$$

and analogously in the leptonic sector. Hence, the above conditions (eq. (5) ) are indeed equivalent to the trend to FD in the up-type and the down-type sectors of quarks and leptons

$$
\triangle_{u}^{(q, l)} \rightarrow 0 \quad \text { and } \quad \triangle_{d}^{(q, l)} \rightarrow 0
$$

\section{Analysis of Yukawa Couplings with RGE's in SM with one and two Higgs Doublets}

\subsection{RGE's of SM for Yukawa Parameters}

The renormalization group equations (RGE's) for coupling parameters tell us how these parameters effectively change when the energy $\mu$ of the experimental probes changes. The 1-loop RGE's of SM for Yukawa parameters have been systematically written down in literature (e. g. []] and references therein), for different versions of SM.

\footnotetext{
${ }^{3}$ Superscript $D$ in $m_{\nu}^{D}$ denotes that these are Dirac neutrino masses; $\eta^{(q)}=\left(V_{c k m}^{(q)}\right)_{c b}$, and $\eta^{(l)}$ is the corresponding element in the leptonic CKM matrix.
} 
In SM with two Higgs doublets (type II) [1], the Yukawa couplings $U_{i j}^{(q)}, D_{i j}^{(q)}, U_{i j}^{(l)}$ and $D_{i j}^{(l)}$ are defined as the couplings contained in the following gauge invariant Lagrangian

$$
\begin{aligned}
\mathcal{L}_{\text {Yukawa }}= & -\sum_{i, j=1}^{3}\left\{\left[\left(\bar{q}_{L}^{(i)} \tilde{H}^{(u)}\right) q_{u R}^{(j)} U_{i j}^{(q)}+\text { h.c. }\right]+\left[\left(\bar{q}_{L}^{(i)} H^{(d)}\right) q_{d R}^{(j)} D_{i j}^{(q)}+\text { h.c. }\right]\right\} \\
& -\sum_{i, j=1}^{3}\left\{\left[\left(\bar{l}_{L}^{(i)} \tilde{H}^{(u)}\right) l_{u R}^{(j)} U_{i j}^{(l)}+\text { h.c. }\right]+\left[\left(\bar{l}_{L}^{(i)} H^{(d)}\right) l_{d R}^{(j)} D_{i j}^{(l)}+\text { h.c. }\right]\right\} .
\end{aligned}
$$

Here we used the notations of eqs. (2) and (3). In the case of the minimal SM, we have to replace $H^{(u)}, H^{(d)} \mapsto H$ in the above form of $\mathcal{L}_{\text {Yukawa }}$.

We can deduce from [7] the corresponding 1-loop RGE's for the Yukawa matrices

$$
Q^{(u)}=U^{(q)} U^{(q) \dagger}, Q^{(d)}=D^{(q)} D^{(q) \dagger}, L^{(u)}=U^{(l)} U^{(l) \dagger}, L^{(d)}=D^{(l)} D^{(l) \dagger} .
$$

These RGE's are of the form

$$
\begin{aligned}
32 \pi^{2} \frac{d}{d t} Q^{(u)} & =3 Q^{(u)^{2}}+\kappa\left(Q^{(u)} Q^{(d)}+Q^{(d)} Q^{(u)}\right)+2 Q^{(u)}\left(\Xi_{u}^{(q)}-A_{u}^{(q)}\right), \\
32 \pi^{2} \frac{d}{d t} Q^{(d)} & =3 Q^{(d)^{2}}+\kappa\left(Q^{(u)} Q^{(d)}+Q^{(d)} Q^{(u)}\right)+2 Q^{(d)}\left(\Xi_{d}^{(q)}-A_{d}^{(q)}\right), \\
32 \pi^{2} \frac{d}{d t} L^{(u)} & =3 L^{(u)^{2}}+\kappa\left(L^{(u)} L^{(d)}+L^{(d)} L^{(u)}\right)+2 L^{(u)}\left(\Xi_{u}^{(l)}-A_{u}^{(l)}\right), \\
32 \pi^{2} \frac{d}{d t} L^{(d)} & =3 L^{(d)^{2}}+\kappa\left(L^{(u)} L^{(d)}+L^{(d)} L^{(u)}\right)+2 L^{(d)}\left(\Xi_{d}^{(l)}-A_{d}^{(l)}\right),
\end{aligned}
$$

where

$$
\begin{gathered}
t=\ln \left(\frac{2 \mu^{2}}{v^{2}}\right) \\
A_{u}^{(q)}=\pi\left[\frac{17}{3} \alpha_{1}+9 \alpha_{2}+32 \alpha_{3}\right], \quad A_{d}^{(q)}=A_{u}^{(q)}-4 \pi \alpha_{1}, \\
A_{u}^{(l)}=\pi\left[3 \alpha_{1}+9 \alpha_{2}\right], \quad A_{d}^{(l)}=\pi\left[15 \alpha_{1}+9 \alpha_{2}\right] .
\end{gathered}
$$

Here, $\alpha_{1}, \alpha_{2}$ and $\alpha_{3}$ are the usual SM gauge couplings corresponding to $U(1)_{Y}, S U(2)_{L}$ and $S U(3)_{c}$, respectively:

$$
\alpha_{i}(\mu)=\alpha_{i}\left(M_{Z}\right)\left[1+\frac{c_{i}}{4 \pi} \alpha_{i}\left(M_{Z}\right) \ln \left(\frac{\mu^{2}}{M_{Z}^{2}}\right)\right]^{-1}
$$

with

$$
\alpha_{1}\left(M_{Z}\right)=0.01013, \quad \alpha_{2}\left(M_{Z}\right)=0.03322, \quad \alpha_{3}\left(M_{Z}\right)=0.109
$$

The other quantities in these equations differ from each other in the two cases of SM 


\begin{tabular}{|c|c|}
\hline SM with two Higgs doublets (type II) & minimal SM \\
\hline$\kappa=\frac{1}{2}$ & $\kappa=-\frac{3}{2}$ \\
$\Xi_{u}^{(q)}=\Xi_{u}^{(l)}=\operatorname{Tr}\left(3 Q^{(u)}+L^{(u)}\right)$ & $\Xi_{u, d}^{(q)}=\Xi_{u, d}^{(l)}=$ \\
$\Xi_{d}^{(q)}=\Xi_{d}^{(l)}=\operatorname{Tr}\left(3 Q^{(d)}+L^{(d)}\right)$ & $\operatorname{Tr}\left(3 Q^{(u)}+3 Q^{(d)}+L^{(u)}+L^{(d)}\right)$ \\
$c_{1}=-7, c_{2}=3, c_{3}=7$ & $c_{1}=-\frac{41}{6}, c_{2}=\frac{19}{6}, c_{3}=7$ \\
\hline
\end{tabular}

Note that the mass matrices are proportional to the VEV's of the Higgses

$$
M_{u}^{(q, l)}=\frac{v_{u}}{\sqrt{2}} U^{(q, l)}, \quad M_{d}^{(q, l)}=\frac{v_{d}}{\sqrt{2}} D^{(q, l)},
$$

where

$$
\begin{gathered}
\left\langle H^{(u)}\right\rangle_{o}=\frac{1}{\sqrt{2}}\left(\begin{array}{c}
0 \\
v_{u}
\end{array}\right), \quad\left\langle H^{(d)}\right\rangle_{o}=\frac{1}{\sqrt{2}}\left(\begin{array}{c}
0 \\
v_{d}
\end{array}\right), \\
v_{u}^{2}+v_{d}^{2}=v^{2}\left(=246^{2} G e V^{2}\right) .
\end{gathered}
$$

For the minimal SM, we replace in the above relations $H^{(u)}, H^{(d)} \mapsto H$ and $v_{u}, v_{d} \mapsto v$. Hence, SM with two Higgs doublets (type II) has one additional, as yet free, parameter $v_{u} / v_{d}$, which crucially influences the running masses. Mathematically, we see this in eq. (14) which implies different boundary conditions in RGE's at $\mu=1 \mathrm{GeV}$ in comparison to the minimal SM. Theoretical bounds on $v_{u} / v_{d}$ have been investigated [8], by requiring that certain processes involving Higgs particles and top quarks are described well by perturbative approach. This results in $v_{u} / v_{d}$ being between 0.1 and 100. On the other hand, experimental evidence from $B-\bar{B}$ mixing, $D-\bar{D}$ mixing, $\triangle m_{K}, \epsilon_{K}$ and missing $E_{T}$ measurements at $p \bar{p}$ colliders suggests more restrictive bounds [8]

$$
0.3<\frac{v_{u}}{v_{d}}<10
$$

\subsection{Numerical Results and Discussions - Quark Sector Only}

First we limit ourselves to the quark sector $-2 \times 2$ matrices corresponding to the two families $(\mathrm{c}, \mathrm{s})$ and $(\mathrm{t}, \mathrm{b})$, taking the leptonic $2 \times 2$ matrices in the RGE's (11) to be zero. In this case, the low energy boundary conditions for the masses of light quarks and the mixing are well known [3, 6], and $m_{t}^{p h y} \sim 100-200 \mathrm{GeV}$. Fig. 1 shows the running of quark masses for a typical value of VEV ratio $v_{u} / v_{d}=1$. The running of the flavor democracy parameters $\delta_{u}^{(q)}\left(=m_{c} / m_{t}\right)$, $\delta_{d}^{(q)}\left(=m_{s} / m_{b}\right)$ and $\eta^{(q)}\left(=\left(V_{c k m}^{(q)}\right)_{c b}\right)$ in this case, and in the case of $v_{u} / v_{d}=0.5$, are depicted in Figs. 2, 3, respectively. These quantities for the minimal SM are depicted in Fig. 4. The ratio 
of the Yukawa couplings $\left(g_{b} / g_{t}\right)$ as function of energy, for various ratios of VEV's, is shown in Fig. 5.

In the minimal SM, quark masses reach their poles $\left(m_{q} \rightarrow \infty\right)$ before the Planck scale only for very heavy top $\left(m_{t}^{p h y} \geq 215 \mathrm{GeV}\right.$ ), as can be seen also from Fig. 4 (for $m_{t}^{p h y}=250 \mathrm{GeV} \Rightarrow$ $\left.\Lambda_{\text {pole }} \approx 10^{10.3} \mathrm{GeV}\right)$. On the other hand, the FD parameters decrease (i. e. positive trend to FD) only in the up-type sector: $\delta_{u}^{(q)}$ decreases, while the parameter $\delta_{d}^{(q)}$ increases always with energy, as well as does the mixing parameter $\eta^{(q)}$. Furthermore, $m_{b} / m_{t} \rightarrow 0$, rather than $m_{b} / m_{t} \rightarrow 1$. The conditions for FD (eq. (7)) are clearly not fulfilled in the minimal SM.

Results for SM with two Higgs doublets (type II), depicted in Figs. 1-3, show a more interesting structure. In all these cases, all the FD parameters $\left(\delta_{u}^{(q)}, \delta_{d}^{(q)}\right.$ and $\left.\eta^{(q)}\right)$ are decreasing functions of energy. Hence, here we have a clear trend to FD (7). Furthermore, in most of these cases, the poles $\Lambda_{\text {pole }}$ for the masses are reached long before $\Lambda_{\text {Planck }}$ : $\log \Lambda_{\text {pole }}<\log \Lambda_{\text {Planck}}$. Table 1 contains $\Lambda_{\text {pole }}$ and $\Lambda_{\text {pert }}$ for various $v_{u} / v_{d}$ and $m_{t}^{p h y} . \Lambda_{\text {pert }}$ is the energy where the perturbative approach mathematically breaks down t. We can interpret these quantities as being of the order of magnitude of the transition energy $\Lambda_{\text {trans }}$ between SM and some extended flavor gauge theory $(\mathrm{FGT}): \log \Lambda_{\text {trans }} \simeq \log \Lambda_{\text {pert }} \simeq \log \Lambda_{\text {pole. }}$. The clear trend to FD at these energies within SM could be interpreted as a signal of a corresponding new physics.

\subsection{Results and Discussions - Leptonic Sector Included}

By including also the leptonic sector in the numerical investigations of the RGE's (11), we were able to investigate also the behavior of the corresponding FD parameters separately in the leptonic sector: $\delta_{u}^{(l)}\left(=m_{\nu_{\mu}}^{D} / m_{\nu_{\tau}}^{D}\right), \delta_{d}^{(l)}\left(=m_{\mu} / m_{\tau}\right)$ and $\eta^{(l)}\left(=\left(V_{c k m}^{(l)}\right)_{\nu_{\mu} \tau}\right)$.

However, for the boundary conditions of RGE's at low energy $(\mu=1 G e V)$ we need the masses of $\mu, \tau, \nu_{\mu}^{\text {Dirac }}$ and $\nu_{\tau}^{\text {Dirac }}$. While the masses of $\mu$ and $\tau$ are well known ( $m_{\mu}^{\text {phy }}=0.106 G e V$, $m_{\tau}^{p h y}=1.78 \mathrm{GeV}$ ), the Dirac neutrino masses are not. However, by invoking the usual "see-saw mechanism" [10] and assuming that $\Lambda_{\text {trans }}\left(\sim \Lambda_{\text {pole }}\right)$ is of the order of magnitude of the Majorana mass $M_{R}$, we can estimate the masses of Dirac neutrinos at low energy (see Section 4 and eq. (19) for more details)

$$
m_{\nu}^{D} \sim \sqrt{M_{R} m_{\nu}^{p h y}} \sim \sqrt{\Lambda_{\text {pole }} m_{\nu}^{p h y}} .
$$

\footnotetext{
${ }^{4}$ It was determined by requiring that the 2-loop correction to $d g_{t} / d t$ in RGE's for Yukawa couplings in the minimal SM 9] be approximately equal at $\Lambda_{\text {pert }}$ to the corresponding 1-loop contribution, resulting in $g_{t}(\mu=$ $\left.\Lambda_{\text {pert }}\right)=\sqrt{6} \pi$. We use this relation also as a reasonable estimate in SM with two Higgs doublets.
} 
Using the upper bounds on $m_{\nu_{\mu}}^{\text {phys }}(<250 \mathrm{keV})$ and $m_{\nu_{\tau}}^{\text {phys }}(<31 \mathrm{MeV})$ as suggested by experiment [11], we obtain for a typical case of $v_{u} / v_{d}=1$ and $m_{t}^{p h y}=200 G e V$ the low energy Dirac neutrino masses $m_{\nu_{\tau}}^{D} \sim 100 \mathrm{GeV}$ and $m_{\nu_{\mu}}^{D} \sim 10 \mathrm{GeV}$. We use these estimated values in the boundary conditions for the RGE's (11) to investigate the FD behavior simultaneously for the quark and the leptonic sector. The FD behavior in the leptonic sector turns out to be completely analogous to that in the quark sector: in the minimal SM $\delta_{d}^{(l)}$ and $\eta^{(l)}$ increase, while in SM with two Higgs doublets (type II) all leptonic FD parameters $\left(\delta_{u}^{(l)}, \delta_{d}^{(l)}\right.$ and $\left.\eta^{(l)}\right)$ decrease with increasing energy and we have a clear trend to FD also in the leptonic sector. In Table 2 we display some of the pertaining results. It is to be noted that the transition energy $\left(\simeq \Lambda_{\text {pole }}\right)$ is in most cases diminished on the logarithmic scale by about 5-10 per cent with the inclusion of the leptonic sector. Furthermore, $m_{b} / m_{t}$ and $m_{\tau} / m_{\nu_{\tau}}^{D}$ decrease with increasing energy.

We have numerically checked also that these conclusions are qualitatively independent of the specific chosen Dirac neutrino masses at low energy $m_{\nu}^{D}(\mu=1 G e V)$.

\section{Top and Neutrino Mass Estimates - under the Assumption of Quark-Lepton Flavor Democracy}

In this section we extend our investigation by imposing, in addition to the conditions (7) for FD in the quark (q-q) and leptonic (l-1) sector, the condition of FD for the (combined) quark-leptonic (q-1) sector

$$
\frac{m_{\nu_{\tau}}^{D}}{m_{t}}, \frac{m_{\tau}}{m_{b}} \rightarrow 1 \quad \text { as } E \rightarrow \Lambda_{\text {trans }} .
$$

Note that this would correspond, in the FGT-scheme of Appendix A, to the assumption that FGT treats quarks and leptons on equal grounds: $\kappa_{\alpha}^{(q)}=\kappa_{\alpha}^{(l)}$, (for $\alpha=u, d$ ) in eq. (A.4). In eq. (3), this would correspond to $G_{\alpha}^{(q)}=G_{\alpha}^{(l)}($ for $\alpha=u, d)$.

In the calculation here, we take into account only the third families $(t, b)$ and $\left(\nu_{\tau}^{D}, \tau\right)$, and investigate the running of their Yukawa couplings (or equivalently: their masses) with increasing energy. It can be checked that the lighter second families do not affect the behavior of the third families in any appreciable way (vice-versa is not true). Here, we approximate, for simplicity, the transition energy $\Lambda_{\text {trans }}$ between SM and an extended theory (e. g. FGT) with $\Lambda_{\text {pole }}$. Such an approximation may look reasonable in view of the fact that we have only the indications for the relation $\log \Lambda_{\text {trans }} \simeq \log \Lambda_{\text {pole }}$. Hence, our additional (high energy) boundary conditions for 
the RGE's will be approximated as

$$
\frac{m_{\nu_{\tau}}^{D}}{m_{t}}=1, \quad \frac{m_{\tau}}{m_{b}}=1 \quad \text { at } E \approx \Lambda_{\text {pole }} .
$$

These conditions are taken into account in our numerical calculations, together with the known low energy boundary conditions $m_{\tau}^{p h y}=1.78 \mathrm{GeV}, m_{b}(\mu=1 \mathrm{GeV})=5.3 \mathrm{GeV}, m_{t}^{p h y}=$ 100, 150, 200, 250GeV. For chosen values of the VEV ratio $v_{u} / v_{d}$ and $m_{t}$, we found the masses of Dirac tau-neutrino $m_{\nu_{\tau}}^{D}$ at $\mu=1 G e V$ which satisfy the above boundary conditions, by using numerical integration of RGE's from $\mu=1 G e V$ to $\Lambda_{\text {pole }}$. For illustration, the resulting curves for one specific case $\left(v_{u} / v_{d}=1\right.$ and $\left.m_{t}^{p h y}=200 \mathrm{GeV}\right)$ are depicted in Fig. 6 . The results for $\Lambda_{\text {trans }}$ (taken as equal to $\Lambda_{\text {pole }}$ ) and $m_{\nu_{\tau}}^{D}$ (at $\mu=1 G e V$ ), for various values of $v_{u} / v_{d}$ and $m_{t}^{p h y}$, are given in Table 3. Since the Dirac neutrino masses shown in Table 3 are too large to be compatible with results of the available experimental predictions [11], we have to invoke the usual "see-saw" mechanism [10] of the mixing of the Dirac neutrino masses (at low energy) and the much larger Majorana neutrino masses $M_{R}$, in order to derive the small physical neutrino mass

$$
m_{\nu}^{p h y} \approx \frac{\left(m_{\nu}^{D}\right)^{2}}{M_{R}}
$$

Since the Majorana mass term breaks the lepton number conservation, the Majorana masses are expected to be of the order of some new (unification) scale $\Lambda\left(\gg \mathcal{O}\left(M_{W}\right)\right)$, and are usually assumed to be $M_{R} \simeq \Lambda$. Within our context, the simplest choice of this new unification scale would be the energy $\Lambda_{\text {trans }}$ where SM is replaced by an extended gauge theory (e. g. an FGT). Since we took here $\Lambda_{\text {trans }} \approx \Lambda_{\text {pole }}$, we obtain

$$
m_{\nu}^{p h y} \approx \frac{\left(m_{\nu}^{D}\right)^{2}}{\Lambda_{\text {pole }}} .
$$

The last entry in Table 3 is calculated according to this equation. As we can see, the physical tauneutrino masses $m_{\nu_{\tau}}^{p h y}$ predicted in this way are very small for the most cases of the choice $v_{u} / v_{d}$ and $m_{t}^{p h y}$, i. e. in most cases they are below the experimentally predicted upper bounds 11.

Note that the scenario in this section, leading to our predictions of $m_{\nu_{\tau}}^{p h y}$, implicitly contains the following assumptions that are not contained in the rest of the paper:

(a) An extended flavor democratic gauge theory (FGT) treats the quark and the leptonic sectors "on equal grounds", i. e. implies the equality of the corresponding quark and leptonic Yukawa couplings near the FGT-SM transition energy $\Lambda_{\text {trans }}$; (b) FGT contains in addition Majorana neutrinos, and its energy range of validity also provides the scale for the heavy Majorana 
masses (i. e. $M_{R} \sim \Lambda_{\text {trans }}$ ); (c) At energies of validity of SM (with two Higgs doublets, type II), we consider that Majorana neutrinos remain decoupled (or very weakly coupled) to the Dirac neutrinos.

In general, we could assume $\mathcal{O}\left(M_{R}\right) \simeq \mathcal{O}\left(\Lambda_{\text {newscale }}\right) \geq \mathcal{O}\left(\Lambda_{\text {pole }}\right)$, and we would consequently end up with even smaller $m_{\nu}^{p h y}$ than those obtained in Table 3.

As seen in Table 3, when increasing $m_{t}^{p h y}$ at a fixed $v_{u} / v_{d}, m_{\nu_{\tau}}^{D}$ at $\mu=1 G e V$ increases and $\Lambda_{\text {pole }}$ decreases, hence $m_{\nu_{\tau}}^{p h y}$ increases. This provides us with upper and lower bounds on the values of $m_{t}^{p h y}$ (at a given $v_{u} / v_{d}$ ) for various specific upper bounds imposed on $m_{\nu_{\tau}}^{p h y}$ (e. g. $\leq 31 \mathrm{MeV}$ [1], or $\leq 1 \mathrm{MeV}$, or $\leq 17 \mathrm{keV}$ [12]) and on $\Lambda_{\text {pole }}$ (e. g. $\leq \Lambda_{\text {Planck }}$, or $\leq 10^{10} \mathrm{GeV}$, or $\left.\leq 10^{5} \mathrm{GeV}\right)$, respectively. These bounds on $m_{t}^{p h y}$ are depicted as functions of $v_{u} / v_{d}$ in Fig. 7 . Even with the largest possible upper bounds on $m_{\nu_{\tau}}^{\text {phy }}(\leq 31 \mathrm{MeV})$ and $\Lambda_{\text {pole }}\left(\leq \Lambda_{\text {Planck }}\right)$, we can still get the narrow bands on the values of $m_{t}^{\text {phy }}$ at any given $v_{u} / v_{d}$. For example, if $v_{u} / v_{d}=1$, then $m_{t}^{p h y}$ ranges between $150 \mathrm{GeV}$ and $215 \mathrm{GeV}$. Inversely, if $m_{t}^{\text {phy }}=150 \mathrm{GeV}$, then $0.5 \leq v_{u} / v_{d} \leq 1.0$.

\section{$5 \quad$ Further Discussions}

The calculations in the paper, except for the previous section, were carried out by including the CKM mixing between the considered second and third families of quarks, by taking $\left(V_{c k m}^{(q)}\right)_{c b}(\mu=$ $1 G e V)=0.05$, i.e. the experimentally suggested value for the quark sector [6]. For simplicity, we took $\left(V_{c k m}^{(l)}\right)_{\nu_{\mu} \tau}(\mu=1 G e V)=0.05$, the same value as in the quark sector. If we ignored any of the flavor mixings (this would imply that the corresponding CKM matrix is the $2 \times 2$ identity matrix at any energy), the results for the masses and the FD parameters $\delta_{u}^{(q, l)}$ and $\delta_{d}^{(q, l)}$ would be minimally changed - in general for about 1 per cent or less, as numerically checked. Furthermore, as argued in section 2 , the lightest fermion families $(u, d)$ and $\left(\nu_{e}^{D}, e\right)$, being practically massless, do not affect the evolution behavior of the other families in any appreciable way (vice-versa is not true) and hence do not affect the conclusions of this paper.

We have not dealt with another possibility of the minimally extended SM's - the ones with two Higgs doublets of which only one contributes to the quark masses (two Higgs doublets, type I [1, [1). Such extensions turn out to have basically equal RGE's in the quark sector as the minimal SM. However, we have in such a case different quark mass relations than in the minimal SM: $g_{q}(\mu)=\sqrt{2} m_{q}(\mu) / v_{1}$, where $v_{1}$ is VEV of the Higgs coupled to the quarks, 
$v_{1}<v=\sqrt{v_{1}^{2}+v_{2}^{2}}$. This would imply that for the same $m_{t}^{p h y}$, the low energy Yukawa coupling $g_{t}(\mu=1 G e V)$ is larger in this model than in the minimal SM. Hence, $\Lambda_{\text {pole }}\left(<\Lambda_{\text {Planck }}\right)$ would exist for a certain range $m_{t}^{p h y} \geq\left(v_{1} / v\right) \times(215 G e V)$, a range including values well below $200 \mathrm{GeV}$, while in the case of the minimal SM $m_{t}^{p h y} \geq 215 \mathrm{GeV}$. However, the running of the other Yukawa couplings (including the leptonic) is, as a rule, very much dominated by the running of $g_{t}$ (and not vice-versa), as in the minimal SM. Among other things, this would imply that $\delta_{d}^{(q)}$ and $\eta^{(q)}$ would again, as in the minimal SM, always increase (away from FD) with increasing energy, as in Fig. 4.

In this paper, we did not consider the Higgs masses and their evolution with energy. We were allowed to ignore them because, unlike the case of the 2-loop RGE's, the 1-loop RGE's for Yukawa couplings do not couple to parameters of the Higgs potential.

The results of the paper, with the view to flavor democracy, advocate a specific extension of the minimal SM - that with two Higgs doublets (type II), where one Higgs doublet couples primarily to up-type fermions $\left(c, t, \nu_{\mu}^{D}\right.$, etc.), and the other to the down-type fermions $(s, b, \mu$, etc.). Note that this extended SM automatically satisfies the relation $\rho \equiv M_{W}^{2} / M_{Z}^{2} \cos ^{2} \theta_{w}=1$ and has no FCNC at the tree level. Motivated by the results of this paper, we express the conjecture that SM with two Higgs doublets (type II), when naturally connected at $E=\Lambda_{\text {trans }}$ with an extended gauge theory (FGT, see also Appendix A), may yield a realistic mechanism for condensation of two Higgses, $\langle\bar{t} t+\ldots\rangle$ and $\langle\bar{b} b+\ldots\rangle$ [13].

Let us emphasize that applying RGE's of SM from low to high energies (and not viceversa), together with the notion of flavor democracy and the related parameters $\delta_{u}^{(\alpha)}, \delta_{d}^{(\alpha)}$ and $\eta^{(\alpha)}(\alpha=q, l)$, was crucial to get within SM some signals of possible new physics. With the exception of the section on the physical neutrino masses, the approach of this paper can also be regarded as conservative and independent of any specific assumptions.

There remain many possibilities to extend and continue the work presented in this paper. We have already mentioned the possible investigation of the running Higgs masses, as well as the possible condensation mechanism for the (two) Higgs doublets which could lead to FD. Other investigations could concentrate on the possibility that an extension of SM (e. g. SUSY, fourthgeneration, etc. ) could become effective in an intermediate energy region between SM- and a new FGT-energy region. Furthermore, several FGT schemes (with various choices of symmetry groups) could be investigated for the regions $E>\Lambda_{\text {trans }}$. 


\section{Conclusion}

We investigated the behavior of Yukawa couplings of SM in order to see whether they show trends to flavor democratic (FD) structures as the energy of the probes increases. We found that the minimal SM (and the closely related SM with two Higgs doublets, type I) does not show such trends in the sector of the down-type fermions $(s, b, \mu, \tau)$ and in the CKM-mixing behavior. On the other hand, we found that SM with two Higgs doublets (type II) clearly shows such trends in all sectors (down-type and up-type fermions), as well as in the CKM-mixing behavior. These results may represent some signals of new physics ("flavor democratic") beyond SM. Then

SM with two Higgs doublets (type II) would be the preferred choice, and $m_{t}^{p h y}$ would be less than $200 G e V$ (for $\Lambda_{\text {trans }} \ll \Lambda_{\text {Planck }}$ ) for most cases of chosen VEV ratio $v_{u} / v_{d}$.

Under the assumption that the corresponding Yukawa couplings in the quark and the leptonic sectors in this model are equal at $E \simeq \Lambda_{\text {trans }}$ (l-q FD), we were able to estimate the masses of top quark and tau-neutrino.

\section{Acknowledgement}

We would like to thank K.J. Abraham, R. Bönisch, H. Lange and E.A. Paschos for helpful discussions. G.C. wishes to thank the Deutsche Forschungsgemeinschaft (DFG) and Dortmund University for financial support during the progress of this work. C.S.K. wishes to thank the German Bundesministerium für Forschung und Technologie (BMFT) for financial support during part of the progress of this work. The work of C.S.K. was also supported in part by the Korean Science and Engineering Foundation, in part by the Center for Theoretical Physics, Seoul National University, and in part by Yonsei University Faculty Research Grant. 


\section{Appendix A Simple Scheme of a "Flavor Gauge Theory"}

Here we briefly introduce a simple scheme for a "flavor gauge theory" (FGT) and point out its relation to the Yukawa sector of SM. The fermionic sector of such an FGT Lagrangian that contains new gauge boson $B_{\mu}$ and gauge coupling $g$ is written schmatically as

$$
\mathcal{L}_{G-f}^{(F G T)}=-g \bar{\Psi} \gamma^{\mu} B_{\mu} \Psi \quad(\text { for } E \geq \Lambda),
$$

where $\Psi$ is the column of all fermions $f_{j}$ (in a usual flavor basis of SM), and $B_{\mu}=B_{\mu}^{j} T_{j}$, with $T_{j}$ being the generator matrices of the (new) symmetry group $G$. For simplicity, we denote here the transition energy $\Lambda_{\text {trans }}$ between FGT and SM as $\Lambda$. We omit the color indices of quarks. The $T_{j}$ 's corresponding to the electrically neutral $B_{\mu}^{j}$ are taken to be proportional to identity matrices in the flavor space, i. e. FGT does not "see" flavors. The effective neutral currentcurrent interaction at "low"-energy $E\left(\Lambda \leq E \ll M_{B}, M_{B}\right.$ being the mass of $\left.B_{\mu}\right)$ can then be written as

$$
\mathcal{L}_{4 f}^{(F G T)} \approx-\frac{g^{2}}{2 M_{B}^{2}} \sum_{i, j}\left(\bar{f}_{i} \gamma^{\mu} f_{i}\right)\left(\bar{f}_{j} \gamma_{\mu} f_{j}\right)
$$

The left-to-right parts of the quark and lepton terms in the above sum are those which can contribute to Yukawa interactions. These parts can be re-expressed, following a suggestion by Bönisch [14], by applying a Fierz transformation [15]

$$
\mathcal{L}_{L-R}^{(F G T)} \approx \frac{2 g^{2}}{M_{B}^{2}} \sum_{i, j}\left[\left(\bar{q}_{i L} q_{j R}\right)\left(\bar{q}_{j R} q_{i L}\right)+\left(\bar{l}_{i L} l_{j R}\right)\left(\bar{l}_{j R} l_{i L}\right)\right] \quad\left(\text { for } \Lambda<E<M_{B}\right) .
$$

We introduce two auxiliary scalar doublets $H^{(u)}$ and $H^{(d)}$ and add to the above Lagrangian the following squares (this transformation doesn't change the path integral for the generating functional, and hence formally doesn't change the physics 16])

$$
\begin{aligned}
\triangle \mathcal{L}= & -\sum_{f=q, l} \sum_{i, j=1}^{3}\left[\kappa_{u}^{(f)} \tilde{H}^{(u) \dagger}+\frac{\sqrt{2} g}{M_{B}} \bar{f}_{L}^{(i)} f_{u R}^{(j)}\right]\left[\kappa_{u}^{(f)} \tilde{H}^{(u)}+\frac{\sqrt{2} g}{M_{B}} \bar{f}_{u R}^{(j)} f_{L}^{(i)}\right] \\
& -\sum_{f=q, l} \sum_{i, j=1}^{3}\left[\kappa_{d}^{(f)} H^{(d) \dagger}+\frac{\sqrt{2} g}{M_{B}} \bar{f}_{L}^{(i)} f_{d R}^{(j)}\right]\left[\kappa_{d}^{(f)} H^{(d)}+\frac{\sqrt{2} g}{M_{B}} \bar{f}_{d R}^{(j)} f_{L}^{(i)}\right],
\end{aligned}
$$

where we used the notations of eq. (2), and $\kappa_{\alpha}^{(q, l)}$ are unspecified masses. Then the Lagrangian (A.3) acquires the form of $\mathcal{L}_{\text {Yukawa }}(\Lambda)$ of eq. (3) (with $G_{\alpha}^{(q, l)}=\kappa_{\alpha}^{(q, l)} g \sqrt{2} / M_{B}$ ) plus bare mass terms of Higgs doublets

$$
\mathcal{L}=\left(\mathcal{L}_{L-R}^{(F G T)}+\triangle \mathcal{L}\right)=\mathcal{L}_{\text {Yukawa }}(\Lambda)-\sum_{\alpha=u, d}\left(\kappa_{\alpha}^{(q) 2}+\kappa_{\alpha}^{(l) 2}\right)\left(H^{(\alpha) \dagger} H^{(\alpha)}\right) .
$$


Several authors (13] and references therein) argued that the auxiliary Higgses in a class of Lagrangians, including this Lagrangian, can become dynamical by quantum effects, becoming basically condensates of the $<\bar{f} f>$-type, when the energy of the probes $E$ is decreased below $\Lambda\left(=\Lambda_{\text {trans }}\right)$ into the SM energy region. The Higgs doublets $H^{(\alpha)}$ then become renormalized by a normalization constant $Z_{\alpha}(E)$ ( which is increasing as $E \uparrow \Lambda$ ), and we arrive at the flavor democratic Yukawa Lagrangian of eqs. (3)-(5) at $E \simeq \Lambda$, with the following substitution

$$
H^{(\alpha)} \mapsto \frac{H^{(\alpha)}}{Z_{\alpha}^{1 / 2}(E)} \Rightarrow G_{\alpha}^{(q, l)}=\frac{\kappa_{\alpha}^{(q, l)} g \sqrt{2}}{M_{B} Z_{\alpha}^{1 / 2}(E)}, \quad(\alpha=u, d)
$$

In such a case, we would end up at low energies with the SM with two Higgs doublets (type II) which tends to flavor democracy (FD) as the energy of the probes increases to the transition energy $\Lambda$. If we expected transition of FGT into the minimal SM, we would have to replace in the above relations

$$
H^{(u)}, H^{(d)} \mapsto H \quad \kappa_{u}^{(q, l)}, \kappa_{d}^{(q, l)} \mapsto \kappa^{(q, l)},
$$

which would lead us to the flavor democratic Yukawa interaction of eq. (1).

We stress that the presented scenario is just a rather primitive scheme of an FGT which may motivate the assumption of the trend to FD in SM as we increase the energy of the probes. There remain many interesting problems connected with such scenarios. One question is how the condensation mechanism works precisely in this case. There is also a question of how to avoid the creation of too many physical Goldstones resulting from the breaking of the horizontal global symmetry $S U(3)_{L} \times S U(3)_{R}$ (-the group of unitary transformations between the three families) 17].

\section{Appendix B Parameters for Deviations from Flavor Democracy}

We define as an FD-flavor basis the flavor basis in which the Yukawa coupling strengths become equal (separately in the up-type and in the down-type sector, see eq. (4)), if the mass spectrum and the mixings are of the form of eq. (5). For simplicity, we will here omit the subscripts and superscripts $(q, l)$ for quarks and leptons, and restrict ourselves to the case of two families $(2 \times 2$ matrices) in a specific (either $q$ or $l$ ) sector.

Transformation of the Yukawa matrices for the up-type and the down-type sectors from the mass basis to a general flavor basis is of the form

$$
U=V_{L}^{u \dagger} U_{\text {diag }} V_{R}^{u}, \quad D=V_{L}^{d \dagger} D_{\text {diag }} V_{R}^{d}
$$


$U_{\text {diag }}$ and $D_{\text {diag }}$ are the mass matrices divided by the corresponding VEV's $(v / \sqrt{2}$ in the case of the minimal SM, and $v_{u, d} / \sqrt{2}$ in the case of SM with two Higgs doublets, type II). $V_{L, R}^{u}$ and $V_{L, R}^{d}$ are orthonormal matrices (the case of no CP-violation assumed) satisfying the relation

$$
V_{L}^{u} V_{L}^{d \dagger}=V_{c k m}
$$

It is straightforward to see that we have a large set of FD-flavor bases

$$
U_{F D}^{(\theta)}=O^{\dagger} R(-\theta) U_{\text {diag }} O, \quad D_{F D}^{(\theta)}=O^{\dagger} R(-\theta) V_{c k m} D_{\text {diag }} O=O^{\dagger} R\left(\theta_{c k m}-\theta\right) D_{\text {diag }} O
$$

where we denoted

$$
O=\frac{1}{\sqrt{2}}\left(\begin{array}{rr}
1 & -1 \\
1 & 1
\end{array}\right), \quad R(-\theta)=\left(\begin{array}{rr}
\cos \theta & -\sin \theta \\
\sin \theta & \cos \theta
\end{array}\right) \quad\left(0 \leq \theta \leq \theta_{c k m}\right),
$$

and

$$
V_{c k m}=\left(\begin{array}{cc}
(1-\epsilon) & \eta \\
-\eta & (1-\epsilon)
\end{array}\right)=R\left(\theta_{c k m}\right) .
$$

For each $\theta$ between zero and $\theta_{c k m}(=\arcsin \eta)$ we have an FD-flavor basis $F D(\theta)$. Taking the usual metrics for the matrices

$$
\|A\|=\left\|\left\{a_{i, j}\right\}\right\|=\sqrt{\sum a_{i j}^{2}},
$$

we can define the deviations from FD for the up-type sector in such a basis as

$$
\triangle_{u, \theta}=\left\|\frac{U_{F D}^{(\theta)}}{\left\|U_{F D}^{(\theta)}\right\|}-\frac{1}{2}\left(\begin{array}{cc}
1 & 1 \\
1 & 1
\end{array}\right)\right\|
$$

and analogously for the down-type sector. Explicit calculations then yield

$$
\begin{gathered}
\triangle_{u, \theta}=\sqrt{2}\left[1-\frac{\cos \theta}{\sqrt{1+\delta_{u}^{2}}}\right]^{1 / 2}=\sqrt{\delta_{u}^{2}+\theta^{2}}\left[1+\mathcal{O}\left(\delta_{u}^{2}, \theta_{c k m}^{2}\right)\right], \\
\triangle_{d, \theta}=\sqrt{2}\left[1-\frac{\cos \left(\theta_{c k m}-\theta\right)}{\sqrt{1+\delta_{d}^{2}}}\right]^{1 / 2}=\sqrt{\delta_{d}^{2}+\left(\theta_{c k m}-\theta\right)^{2}}\left[1+\mathcal{O}\left(\delta_{d}^{2}, \theta_{c k m}^{2}\right)\right],
\end{gathered}
$$

where we denoted $\delta_{u}=m_{c} / m_{t}, \delta_{d}=m_{s} / m_{b}$ for the quark sector, and analogously for the leptonic sector.

Choosing the "up-down symmetric" FD-flavor basis $\left(\theta=\theta_{c k m} / 2 \simeq \eta / 2\right)$, we obtain

$$
\triangle_{u} \simeq \sqrt{\delta_{u}^{2}+\frac{\eta^{2}}{4}}, \quad \triangle_{d} \simeq \sqrt{\delta_{d}^{2}+\frac{\eta^{2}}{4}} .
$$


This apears to be the most reasonable choice to parametrize deviations from FD in the up-type and the down-type sectors (for quarks, and for leptons). On the other hand, various choices of $\theta$ $\left(0 \leq \theta \leq \theta_{c k m}\right)$ would give us similar values for deviations. All these parameters are, of course, functions of the running energy. 


\section{References}

[1] N.G. Deshpande and E. Ma, Phys. Rev. D18 (1978) 2574; J.F. Donoghue and L.-F. Li, Phys. Rev. D19 (1979) 945; L.J. Hall and M.B. Wise, Nucl. Phys. B187 (1981) 397

[2] C.T. Hill, Phys. Rev. D24 (1981) 691; E.A. Paschos, Z. Phys. C26 (1984) 235; J.H. Halley, E.A. Paschos and H. Usler, Phys. Lett. B155 (1985) 107

[3] J. Gasser and H. Leutwyler, Phys. Rep. 87 (1982) 77; S. Narison, Phys. Lett. B197 (1987) 405

[4] CDF Collaboration: F. Abe et al., Fermilab-Pub-91-352-E (Dec. 1991)

[5] J. Ellis and G.L. Fogli, Phys. Lett. B249 (1990) 543; G. Altarelli, R. Barbieri and S. Jadach, Nucl. Phys. B369 (1992) 3; P. Langacker, talk given at Madison SSC Workshop (March 1992)

[6] Particle Data Group: G.P. Yost et al., Phys. Lett. B239 (1990) 1 (Erratum: Phys. Lett. B253 (1991) 524)

[7] C.T. Hill, C.N. Leung and S. Rao, Nucl. Phys. B262 (1985) 517

[8] V. Barger, J.L. Hewett and R.J.N. Phillips, Phys. Rev. D41 (1990) 3421

[9] M.E. Machacek and M.T. Vaughn, Nucl. Phys. B249 (1985) 70; M. Fischer and J. Oliensis, Phys. Lett. B119 (1982) 385; D.R.T. Jones, Phys. Rev. D25 (1982) 581 I. Jack and H. Osborn, Nucl. Phys. B249 (1985) 472

[10] T. Yanagida, Proceedings of the Workshop on Unified Theory and Baryon 3Number of the Universe (KEK, Japan, 1979); M. Gell-Mann, P. Ramond and R. Slansky, in "Supergravity", edited by P. Van Nieuwenhuizen and D.Z. Freedman (North-Holland, Amsterdam, 1979)

[11] ARGUS Collaboration: H. Albrecht et al., Phys. Lett. B202 (1988) 149, B292 (1992) 221;

T.K. Kuo and J. Pantaleone, Rev. of Mod. Phys., Vol. 61, No. 4 (1989) 937

[12] A. Hime, R.J.N. Phillips, G.G. Ross and S. Sankar, Phys. Lett. B260 (1991) 381

[13] M. Suzuki, Phys.Rev. D41 (1990) 3457; M. Harada and N. Kitazawa, Phys. Lett. B257 (1991) 383 
[14] R. Bönisch, Munich preprint LMU-91/03 and private communication

[15] M.A. Shifman, A.I. Vainshtein and V.I. Zakharov, Nucl. Phys. B120 (1977) 316

[16] D. Bailin and A. Love, "Introduction to Gauge Field Theory" (Adam Hilger, Bristol and Boston, 1986); T. Eguchi, Phys. Rev. D14 (1976) 2755

[17] Z. Berezhiani, private communication 


\section{Tables}

Table 1

\begin{tabular}{|c|c|l|l|}
\hline$v_{u} / v_{d}$ & $m_{t}^{\text {phy }}$ & $\Lambda_{\text {pert }}$ & $\Lambda_{\text {pole }}$ \\
\hline \multirow{4}{*}{0.5} & 100 & $(3.16) \cdot 10^{17}$ & $(4.26) \cdot 10^{17}$ \\
\cline { 2 - 4 } & 150 & $(1.20) \cdot 10^{5}$ & $(1.61) \cdot 10^{5}$ \\
\cline { 2 - 4 } & 200 & $(3.80) \cdot 10^{3}$ & $(5.11) \cdot 10^{3}$ \\
\cline { 2 - 4 } & 250 & $(1.20) \cdot 10^{3}$ & $(1.70) \cdot 10^{3}$ \\
\hline \multirow{5}{*}{1.0} & 100 & $>\Lambda_{\text {Planck }}$ & $>\Lambda_{\text {Planck }}$ \\
\cline { 2 - 4 } & 150 & $>\Lambda_{\text {Planck }}$ & $>\Lambda_{\text {Planck }}$ \\
\cline { 2 - 4 } & 200 & $(1.84) \cdot 10^{7}$ & $(2.48) \cdot 10^{7}$ \\
\cline { 2 - 4 } & 250 & $(6.73) \cdot 10^{4}$ & $(9.08) \cdot 10^{4}$ \\
\hline \multirow{5}{*}{5.0} & 100 & $>\Lambda_{\text {Planck }}$ & $>\Lambda_{\text {Planck }}$ \\
\cline { 2 - 4 } & 150 & $>\Lambda_{\text {Planck }}$ & $>\Lambda_{\text {Planck }}$ \\
\cline { 2 - 4 } & 200 & $>\Lambda_{\text {Planck }}$ & $>\Lambda_{\text {Planck }}$ \\
\cline { 2 - 4 } & 250 & $(3.88) \cdot 10^{9}$ & $(5.22) \cdot 10^{9}$ \\
\hline \multirow{5}{*}{ min. SM } & 100 & $>\Lambda_{\text {Planck }}$ & $>\Lambda_{\text {Planck }}$ \\
\cline { 2 - 4 } & 150 & $>\Lambda_{\text {Planck }}$ & $>\Lambda_{\text {Planck }}$ \\
\cline { 2 - 4 } & 200 & $>\Lambda_{\text {Planck }}$ & $>\Lambda_{\text {Planck }}$ \\
\cline { 2 - 4 } & 250 & $(1.5) \cdot 10^{10}$ & $(2.0) \cdot 10^{10}$ \\
\hline
\end{tabular}

Table 1: $\Lambda_{\text {pole }}$ and $\Lambda_{\text {pert }}$ for various $m_{t}^{p h y}$ and for various VEV ratios $v_{u} / v_{d}$ (in SM with two Higgs doublets, type II) and for the the minimal SM. $\Lambda_{\text {pole }}$ is the energy where the quark masses become infinite, $\Lambda_{\text {pert }}$ the energy where the perturbative approach breaks down. The masses of leptons were taken to be zero. All energies are in $\mathrm{GeV} . \Lambda_{\text {Planck }} \simeq 10^{19} \mathrm{GeV}$. 
Table 2

\begin{tabular}{|c|c|c||l|l||l|l|l|l|l|}
\hline$v_{u} / v_{d}$ & $m_{t}^{\text {phy }}$ & $\Lambda_{\text {pole }}$ & $\left(\delta_{u}^{(q)}\right)^{(0)}$ & $\delta_{u}^{(q)}$ & $\delta_{d}^{(q)}$ & $\delta_{u}^{(l)}$ & $\delta_{d}^{(l)}$ & $\eta^{(q)}$ & $\eta^{(l)}$ \\
\hline \multirow{3}{*}{0.5} & 100 & $(3.6) \cdot 10^{5}$ & 0.0113 & 0.0090 & 0.031 & 0.048 & 0.047 & 0.046 & 0.039 \\
\cline { 2 - 9 } & 150 & $(1.1) \cdot 10^{4}$ & 0.0093 & 0.0065 & 0.029 & 0.061 & 0.051 & 0.044 & 0.042 \\
\cline { 2 - 9 } & 200 & $(2.3) \cdot 10^{3}$ & 0.0086 & 0.0058 & 0.029 & 0.070 & 0.053 & 0.044 & 0.044 \\
\hline \multirow{3}{*}{1.0} & 100 & $(1.0) \cdot 10^{18}$ & 0.0095 & 0.0081 & 0.031 & 0.030 & 0.040 & 0.047 & 0.033 \\
\cline { 2 - 9 } & 150 & $(4.1) \cdot 10^{10}$ & 0.0071 & 0.0047 & 0.029 & 0.044 & 0.045 & 0.044 & 0.038 \\
\cline { 2 - 9 } & 200 & $(2.5) \cdot 10^{6}$ & 0.0061 & 0.0035 & 0.027 & 0.070 & 0.053 & 0.041 & 0.044 \\
\hline \multirow{2}{*}{5.0} & 200 & $(1.6) \cdot 10^{16}$ & 0.0052 & 0.0028 & 0.027 & 0.045 & 0.046 & 0.041 & 0.038 \\
\hline \multirow{2}{*}{$\operatorname{minSM}$} & 200 & $(2.8) \cdot 10^{17}$ & 0.0051 & 0.0029 & 0.059 & 0.043 & 0.138 & 0.089 & 0.119 \\
\cline { 2 - 9 } & 250 & $(1.4) \cdot 10^{9}$ & 0.0045 & 0.0023 & 0.065 & 0.077 & 0.077 & 0.100 & 0.065 \\
\hline
\end{tabular}

Table 2: Quark and lepton flavor democracy (FD) parameters at $\mu=\Lambda_{\text {pole }} / 2$, and values of $\Lambda_{\text {pole }}$ (in $G e V$ ), for various $v_{u} / v_{d}$ (in SM with two Higgs doublets, type II), and for the minimal SM, and for various $m_{t}^{p h y}$. The Dirac neutrino masses at $\mu=1 G e V$ were taken to be $m_{\nu_{\mu}}^{D}=10 \mathrm{GeV}$ and $m_{\nu_{\tau}}=100 \mathrm{GeV} .\left(\delta_{u}^{(q)}\right)^{(0)}$ is the value of this parameter at $\mu=1 \mathrm{GeV}$. Other values of the FD parameters at $\mu=1 G e V$ are in this case: $\left(\delta_{d}^{(q)}\right)^{(0)}=0.033 ;\left(\delta_{u}^{(l)}\right)^{(0)}=0.100$; $\left(\delta_{d}^{(l)}\right)^{(0)}=0.060 ;\left(\eta^{(q)}\right)^{(0)}=0.05\left(\left(\eta^{(l)}\right)^{(0)}\right.$ is assumed to be 0.05 , for simplicity). The entries with $m_{t}^{\text {phy }}=100,150 \mathrm{GeV}$ that are missing have $\Lambda_{\text {pole }}>\Lambda_{\text {Planck }}$. 
Table 3

\begin{tabular}{|c|c|c|r|c|}
\hline$v_{u} / v_{d}$ & $m_{t}^{\text {phy }}$ & $\Lambda_{\text {pole }}$ & $m_{\nu_{\tau}}^{D}$ & $m_{\mu_{\tau}}^{\text {phy }}$ \\
\hline \multirow{5}{*}{0.5} & 100 & $(3.20) \cdot 10^{13}$ & 43.04 & $(5.80) \cdot 10^{-11}$ \\
\cline { 2 - 5 } & 150 & $(3.85) \cdot 10^{4}$ & 79.02 & $(1.62) \cdot 10^{-1}$ \\
\cline { 2 - 5 } & 200 & $(2.72) \cdot 10^{3}$ & 93.02 & 3.18 \\
\cline { 2 - 5 } & 250 & $(1.18) \cdot 10^{3}$ & 99.06 & 8.28 \\
\hline \multirow{5}{*}{1.0} & 100 & $>\Lambda_{\text {Planck }}$ & & \\
\cline { 2 - 5 } & 150 & $(6.78) \cdot 10^{16}$ & 60.28 & $(5.36) \cdot 10^{-14}$ \\
\cline { 2 - 5 } & 200 & $(1.95) \cdot 10^{6}$ & 104.47 & $(5.60) \cdot 10^{-3}$ \\
\cline { 2 - 5 } & 250 & $(2.76) \cdot 10^{4}$ & 127.20 & $(5.88) \cdot 10^{-1}$ \\
\hline \multirow{5}{*}{5.0} & 100 & $>\Lambda_{\text {Planck }}$ & & \\
\cline { 2 - 5 } & 150 & $>\Lambda_{\text {Planck }}$ & & \\
\cline { 2 - 5 } & 200 & $(2.50) \cdot 10^{20}$ & 75.22 & $(2.26) \cdot 10^{-17}$ \\
\cline { 2 - 5 } & 250 & $(1.13) \cdot 10^{8}$ & 126.00 & $(1.40) \cdot 10^{-4}$ \\
\hline
\end{tabular}

Table 3: Predictions of $\Lambda_{\text {pole }}, m_{\nu_{\tau}}^{D}$ and $m_{\nu_{\tau}}^{p h y}$ for various values of $v_{u} / v_{d}$ and $m_{t}^{p h y}$ (all energies are in $G e V$ ). For $m_{\nu_{\tau}}^{\text {phy }}$ we used the usual see-saw mechanism with $M_{R} \approx \Lambda_{\text {pole }}$ (eqs. (19), (20)). 


\section{Figure Captions}

Fig. 1: Quark masses as functions of energy scale $\mu$, in SM with two Higgs doublets (type II), for a typical value of VEV ratio $v_{u} / v_{d}=1$. The masses of light quarks $\left(m_{b}, m_{c}, m_{s}\right)$ and mixings at $\mu=1 \mathrm{GeV}$ are fixed by experiment [3, 6], and $m_{t}^{p h y}\left(=m_{t}\left(\mu=m_{t}\right)\right)=100,150,200,250 \mathrm{GeV}$. Leptons were assumed massless.

Figs. 2, 3: Quark flavor democracy parameters $\delta_{u}^{(q)}, \delta_{d}^{(q)}$ and $\eta^{(q)}=\left(V_{c k m}^{(q)}\right)_{c b}$ as functions of energy scale $\mu$, for VEV ratios $v_{u} / v_{d}=1,0.5$, respectively (in SM with two Higgs doublets, type II). Other explanations as for Fig. 1.

Fig. 4: Quark flavor democracy parameters $\delta_{u}^{(q)}, \delta_{d}^{(q)}$ and $\eta^{(q)}$ in the minimal SM. Other explanations as in Figs. 1-3.

Fig. 5: The ratio $g_{b} / g_{t}$ of Yukawa couplings as a function of energy scale, for $m_{t}^{p h y}=200 \mathrm{GeV}$ and VEV ratios $v_{u} / v_{d}=0.5,1.0,5.0$ (in SM with two Higgs doublets, type II).

Fig. 6: Masses of $\tau, b, \nu_{\tau}^{\text {Dirac }}$ and $t$ as functions of energy scale $\mu$, for $m_{t}^{\text {phy }}=200 G e V$ and $v_{u} / v_{d}=1$ (in SM with two Higgs doublets, type II). The masses $m_{b}$ and $m_{\tau}$ at $\mu=1 G e V$ are fixed by experiments. The Dirac $\nu_{\tau}$ masses at $\mu=1 \mathrm{GeV}$, which satisfy all the boundary conditions at $\mu=1 \mathrm{GeV}$ and the high energy boundary conditions (18), were found by numerical integration of RGE's from $\mu=1 G e V$ to $\Lambda_{\text {pole }}$.

Fig. 7: Upper and lower bounds on the values of $m_{t}^{p h y}$ as functions of $v_{u} / v_{d}$ for various specific upper bounds imposed on $m_{t}^{p h y}(e . g . \leq 31 \mathrm{MeV}$, or $\leq 1 \mathrm{MeV}$, or $\leq 17 \mathrm{keV})$ and on $\Lambda_{\text {pole }}$ (e. g. $\leq \Lambda_{\text {Planck }}$, or $\leq 10^{10} \mathrm{GeV}$, or $\leq 10^{5} \mathrm{GeV}$ ), respectively. 